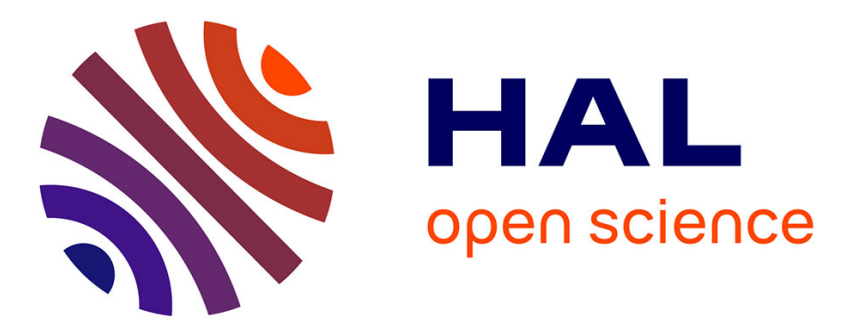

\title{
Constraining Fault Friction and Stability With Fluid-Injection Field Experiments
}

Stacy Larochelle, Nadia Lapusta, Jean Paul Ampuero, Frédéric Cappa

\section{To cite this version:}

Stacy Larochelle, Nadia Lapusta, Jean Paul Ampuero, Frédéric Cappa. Constraining Fault Friction and Stability With Fluid-Injection Field Experiments. Geophysical Research Letters, 2021, 48 (10), pp.e2020GL091188. 10.1029/2020gl091188 . hal-03246984

\section{HAL Id: hal-03246984 \\ https://hal.science/hal-03246984}

Submitted on 3 Jun 2021

HAL is a multi-disciplinary open access archive for the deposit and dissemination of scientific research documents, whether they are published or not. The documents may come from teaching and research institutions in France or abroad, or from public or private research centers.
L'archive ouverte pluridisciplinaire HAL, est destinée au dépôt et à la diffusion de documents scientifiques de niveau recherche, publiés ou non, émanant des établissements d'enseignement et de recherche français ou étrangers, des laboratoires publics ou privés. 


\section{Geophysical Research Letters}

\author{
RESEARCH LETTER \\ 10.1029/2020GL091188 \\ Key Points: \\ - Multiple frictional models with \\ different stability reproduce the slip \\ observed during the pressurization \\ stage of a field experiment \\ - The depressurization phase \\ provides additional constraints on \\ hydromechanical parameters and \\ hence fault stability \\ - Fault stability and the spatial exten \\ of slip relative to the pressurized \\ region depend on residual friction \\ versus initial stress levels
}

Supporting Information: Supporting Information may be found in the online version of this article.

Correspondence to:

S. Larochelle,

stacy.larochelle@caltech.edu

Citation:

Larochelle, S., Lapusta, N., Ampuero, J.-P., \& Cappa, F. (2021). Constraining fault friction and stability with fluidinjection field experiments. Geophysical Research Letters, 48, e2020GL091188. https://doi.org/10.1029/2020GL091188

Received 7 OCT 2020

Accepted 5 MAY 2021
(C) 2021. American Geophysical Union. All Rights Reserved.

\section{Constraining Fault Friction and Stability With Fluid- Injection Field Experiments}

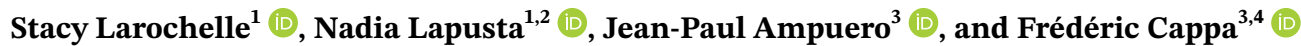 \\ ${ }^{1}$ Division of Geological and Planetary Sciences, California Institute of Technology, Pasadena, CA, USA, ${ }^{2}$ Division of \\ Engineering and Applied Science, California Institute of Technology, Pasadena, CA, USA, ${ }^{3}$ IRD, CNRS, Observatoire de \\ la Côte d'Azur, Université Côte d'Azur, Géoazur, Sophia Antipolis, Nice, France, ${ }^{4}$ Institut Universitaire de France, Paris, \\ France
}

Abstract While the notion that injecting fluids into the subsurface can reactivate faults by reducing frictional resistance is well established, the ensuing evolution of the slip is still poorly understood. What controls whether the induced slip remains stable and confined to the fluid-affected zone or accelerates into a runaway earthquake? Are there observable indicators of the propensity to earthquakes before they happen? Here, we investigate these questions by modeling a unique fluid-injection experiment on a natural fault with laboratory-derived friction laws. We show that a range of fault models with diverging stability with sustained injection reproduce the slip measured during pressurization. Upon depressurization, however, the most unstable scenario departs from the observations, suggesting that the fault is relatively stable. The models could be further distinguished with optimized depressurization tests or spatially distributed monitoring. Our findings indicate that avoiding injection near low-residual-friction faults and depressurizing during slip acceleration could help prevent large-scale earthquakes.

Plain Language Summary Fluid injections into the Earth's crust are common practice in the exploitation of subsurface energy resources such as geothermal energy, shale gas, and conventional hydrocarbons. These injections can perturb nearby fault structures and hence induce earthquakes and transient slow slip. Understanding what controls the stability (i.e., the propensity to generate earthquakes) and spatial extent of the fault response as well as identifying precarious faults is crucial to minimize the seismic hazard associated with these industrial practices. Here, we take a step toward this goal by modeling a unique experiment, in which water was injected into a natural fault and the resulting slip measured directly at depth. We first show that multiple models can explain the observations equally well, while pressure is increased in the experiment. In these models, how stable the fault response is with further injection and how large of a zone is reactivated compared to the fluid-affected region depends on frictional properties. We then demonstrate that the slow slip response to a decrease in injection pressure further constrains the range of admissible models. Our work suggests that it may be possible to identify potentially hazardous faults with optimally designed injection tests without inducing damaging earthquakes.

\section{Introduction}

Earthquakes induced by fluid injection into the subsurface pose a major challenge for the geoenergy industry and society in general (Ellsworth, 2013; Grigoli et al., 2017). Tectonically quiescent regions, where dormant faults could be reactivated are particularly challenging, as their infrastructure is often not designed for large-magnitude induced earthquakes (McGarr et al., 2015). At the same time, some faults have been observed to slip stably at aseismic speeds of $10^{-7}-10^{-2} \mathrm{~m} / \mathrm{s}$, in response to fluid injection (Cornet et al., 1997; Duboeuf et al., 2017; Guglielmi et al., 2015; Scotti \& Cornet, 1994; Wei et al., 2015). While induced earthquakes have been located anywhere from a few meters to tens of kilometers from injection wells (Goebel \& Brodsky, 2018), the spatial extent of fluid-induced aseismic slip is not as well characterized due to the paucity of direct observations. Understanding what conditions lead to seismic versus aseismic and localized versus widespread fault reactivation is central to physics-based hazard forecasting.

An outstanding opportunity to investigate these questions is offered by a decametric-scale fluid injection experiment recently conducted in an underground tunnel intercepting a dormant fault in a carbonate 
A. Field Experiment Setup

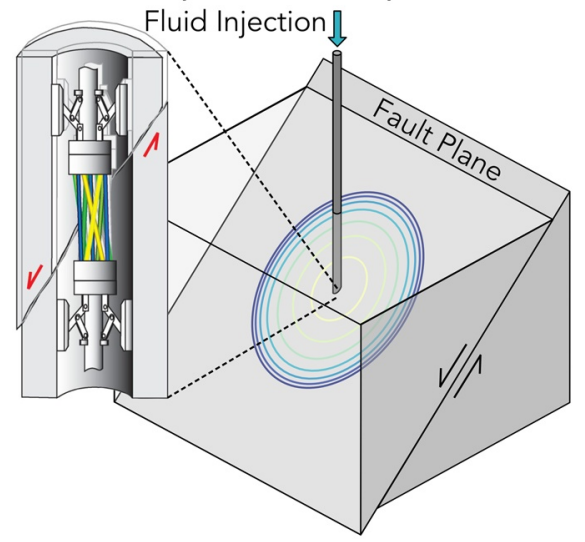

C. Numerical Model

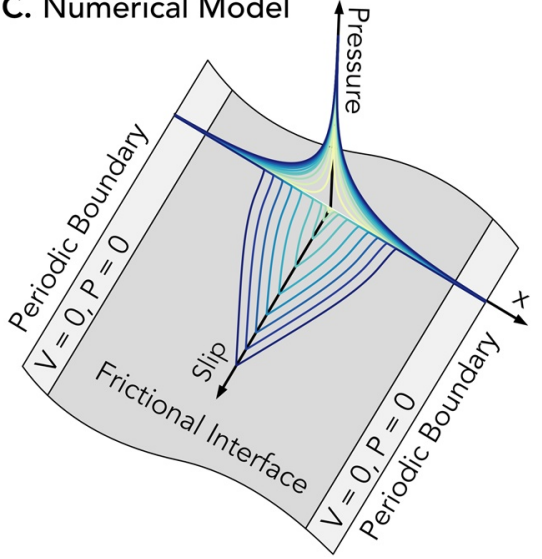

B. Field Experiment Observations
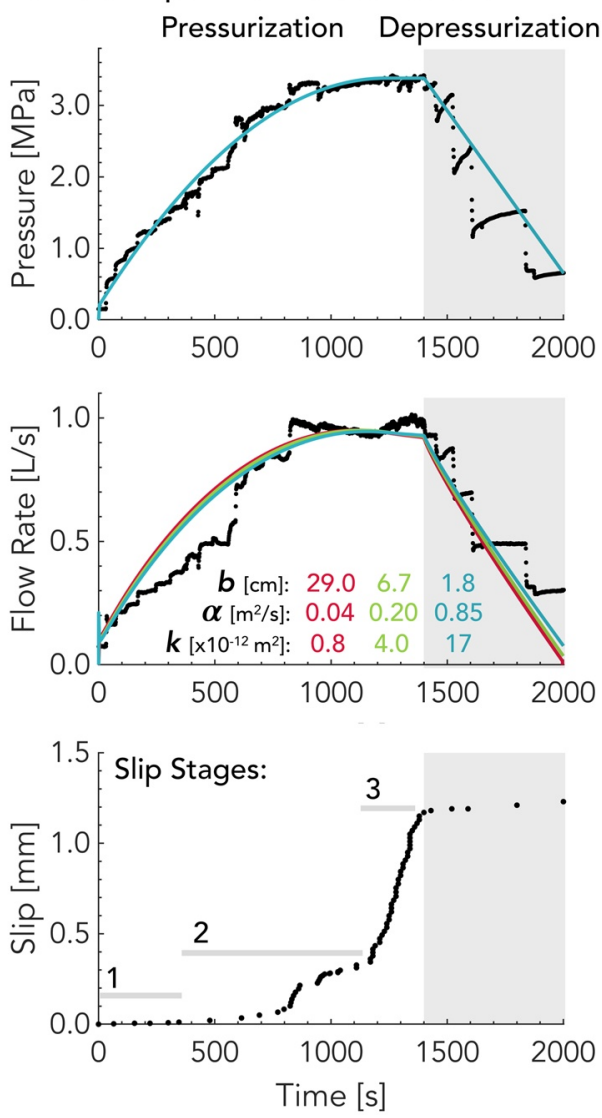

Figure 1. In situ measurement and modeling of fault slip induced by fluid injection. (a) Schematic of the field experiment presented in Guglielmi et al. (2015) in which fluid injected into a borehole crossing a natural but inactive fault caused its reactivation. A special borehole probe (SIMFIP) was used to measure the fault displacements directly at the injection site. (b) Pressure, flow rate, and fault slip measured during the field experiment. The colored lines and associated parameters correspond to the three different hydrological models considered in this study. The gray area indicates the depressurization stage that has not been shown nor modeled in prior studies. (c) Schematic of the model used to simulate slip on a fault plane embedded in an elastic bulk medium. Snapshots of a sample fluid pressure diffusion scenario and its resulting fault slip are shown for illustration (the darker colors indicate later times). Schematics (a) and (c) are not to scale.

formation (Guglielmi et al., 2015) (Figure 1a). During the experiment, the fluid pressure and fault slip were recorded at the injection site. Although the observed slip was mostly aseismic, it is important to understand if the observations contained sufficient information to determine whether slip would have accelerated into an earthquake rupture if injection had continued. Previous efforts to model the field experiment with a slip-weakening friction law concluded that aseismic slip outgrew the pressurized zone, potentially leading to a runaway earthquake with continued injection (Bhattacharya \& Viesca, 2019).

Here, we use the data from the field experiment to examine the issue of slow and confined versus fast and runaway slip in models with more realistic, laboratory-derived rate-and-state friction laws (Dieterich, 1979, 2007; Ruina, 1983) consistent with laboratory results on materials from this specific fault zone (Cappa et al., 2019). Furthermore, we use the modeling to identify promising avenues to quantify the fault properties and control injection-induced seismicity hazard. We adopt a fully dynamic computational framework that resolves both aseismic and seismic slip on faults. We keep other model ingredients relatively simple to better understand frictional effects in the presence of a diffusing fluid. For example, we do not explicitly model the change in fault permeability induced by slip as in previous studies (Bhattacharya \& Viesca, 2019; Cappa et al., 2019; Guglielmi et al., 2015). Nonetheless, we find that multiple frictional scenarios of varying spatial behavior and proneness to large earthquakes match the slip observations of the field 
experiment equally well during fault pressurization. We also find that depressurization provides further constraints that could help identify potentially hazardous faults.

\section{Data and Methods}

\subsection{A Unique Fluid-Injection Experiment on a Natural Fault}

The unprecedented field experiment involved injecting water directly into the fault zone and measuring the resulting fault slip at a depth of $280 \mathrm{~m}$ with a specially designed borehole probe (Guglielmi et al., 2015) (Figure 1a). Prior to the experiment, the shear and normal stress acting on the fault were estimated at $1.65 \pm 0.5$ and $4.25 \pm 0.5 \mathrm{MPa}$, and the permeability and bulk modulus of the initially dry fault at $7 \times 10^{-12} \mathrm{~m}^{2}$ and $13.5 \pm 3.5 \mathrm{GPa}$, respectively. Figure $1 \mathrm{~b}$ summarizes the main observations of the experiment, including the deceleration of slip associated with depressurization not discussed in previous works. The slip measured during the pressurization phase displays three distinct slip stages. At first, the fault is inactive and no significant slip is recorded. The second stage initiates between 300 and $400 \mathrm{~s}$, when slip rates attain $\sim 10^{-7} \mathrm{~m} / \mathrm{s}$ and the accumulated slip becomes measurable within the timeframe of the experiment. Stage 3 corresponds to the sharp acceleration to slip velocities of $\sim 10^{-6} \mathrm{~m} / \mathrm{s}$ without any significant increase in injection pressure at 1200 s. Hydromechanical modeling suggests that $70 \%$ of the 20 -fold increase in permeability during the experiment occurred prior to this acceleration (Guglielmi et al., 2015). Laboratory experiments were also performed on grinded materials from the fault zone to further constrain the rate-and-state frictional properties (Cappa et al., 2019).

\subsection{Diffusion of Pore Fluid Pressure Into the Fault Zone}

We model the field experiment as a fluid injection into a planar fault embedded in an elastic medium (Figures 1a and 1c). We simulate the fluid injection by prescribing an evolution of pore pressure at the center of the fault that approximates the pressure history of the field experiment (Figure 1b, top). Simulations with a smooth pressure evolution result in similar but easier to interpret simulation results than those with the exact pressure history (Figures S1-S2).

The imposed pressure diffuses axisymmetrically into the fault plane as follows:

$$
\frac{\partial p(r, t)}{\partial t}=\alpha\left(\frac{\partial^{2} p(r, t)}{\partial r^{2}}+\frac{1}{r} \frac{\partial p(r, t)}{\partial r}\right)
$$

where $p$ is the pore pressure, $r$ is radial distance, $t$ is time, and $\alpha$ is the hydraulic diffusivity. The diffusion is numerically implemented using a forward finite difference scheme. Injection pressure is prescribed at a distance of $r_{\text {inj }}=0.05 \mathrm{~m}$ from the center of the fault to mimic the experimental procedure. Although we prescribe zero pressure boundary conditions far from the injection point to emulate the initially dry fault, the choice of boundary condition is not essential here because the size of the simulated fault $(250 \mathrm{~m})$ is larger than that of the pressure diffusion. Models with larger fault domains produce nearly identical results (Figure S3).

Although both pressure and flow rate are reported as part of the field experiment, the exact value of the hydraulic diffusivity $\alpha$ is still uncertain because the spatial extent of the pressurized zone and the fault thickness over which the diffusion occurs, $b$, are poorly constrained. The volumetric flow rate, $Q$, depends on $\alpha$ and $b$ as:

$$
Q(t)=-\frac{2 \pi r_{\mathrm{inj}} b \alpha S_{s}}{\rho g} \frac{\partial p}{\partial r}\left(r_{\mathrm{inj}}, t\right)
$$

where $S_{S}$ is the specific storage and $\rho$ the density of water. Hence, for a given flow rate, there is a trade-off between the fault thickness $b$ over which the fluid diffusion occurs, the hydraulic diffusivity $\alpha$ and the specific storage $S_{S}$ of the fault zone (and hence permeability $k=\frac{\alpha S_{S} \eta}{\rho g}$ where $\eta$ is the dynamic viscosity of water). Note that $\alpha$ affects $Q(t)$ via both the prefactor and the $\frac{\partial p}{\partial r}\left(r_{\text {inj }}, t\right)$ term in Equation 2 . In Section 3, 
we use hydraulic diffusivities of $0.04,0.20$, and $0.85 \mathrm{~m}^{2} / \mathrm{s}$ to match field experimental measurements of slip for different friction regimes. Assuming a specific storage of $S_{S}=2 \times 10^{-4} \mathrm{~m}^{-1}$ as in Bhattacharya and Viesca (2019), for example, these hydraulic diffusivities correspond to permeability values of 0.8 , 4, and $17 \times 10^{-12} \mathrm{~m}^{2}$ that are within the ranges presented in previous studies that considered permeability enhancement: $0.8-1.3 \times 10^{-12} \mathrm{~m}^{2}$ (Bhattacharya \& Viesca, 2019) and 7-100 × 10 $0^{-12} \mathrm{~m}^{2}$ (Guglielmi et al., 2015). These permeability values are also consistent with the flow rates measured in the field experiment, for reasonable values of the fault thickness $b$ of $29,6.7$, and $1.8 \mathrm{~cm}$, respectively (Figure $1 \mathrm{~b}$ ). While considering permeability enhancement may be necessary to match the finer features of the pressure and flow rate histories (unless the fault thickness $b$ affected by fluid flow varies with time or with space), all three combinations of the parameters we use reproduce the hydrologic observations to the first order. We, therefore, consider a range of constant hydraulic diffusivity (and hence permeability) values in our search for models that reproduce the main features of the experimental observations.

\subsection{Numerical Modeling of Fluid-Induced Fault Slip}

As fluid pressure increases and diffuses into the fault plane, fault friction eventually decreases and measurable slip ensues (Figure 1c). We model this induced fault slip using a fully dynamic 2D antiplane boundary integral method capable of simulating the complete seismic cycle including both aseismic and seismic deformation (Lapusta et al., 2000; Noda \& Lapusta, 2013). Fault slip is governed by the following elastodynamic equation:

$$
\tau(x, t)=f[\sigma-p(x, t)]=\tau_{\text {ini }}+F(\delta(x, t))-\frac{\mu}{2 c_{s}} V(x, t)
$$

where $\tau$ is the shear stress, $f$ the friction coefficient, $\sigma$ the normal stress, $\tau_{\text {ini }}$ the initial (i.e., background) shear stress, $F$ a linear functional which depends on the slip history, $\delta$, $\mu$ the shear modulus of the elastic medium, $c_{s}$ the shear wave speed, and $V$ the slip rate. The friction coefficient in Equation 3 follows an empirical rate-and-state formulation derived from laboratory experiments which describes the dependence of $f$ on the slip rate and a state variable $\theta$ (Dieterich, 1979, 2007; Ruina, 1983):

$$
f(V, \theta)=f^{*}+a \ln \frac{V}{V^{*}}+b \ln \frac{V^{*} \theta}{D_{\mathrm{RS}}}
$$

where $a$ and $b$ are the direct and evolutionary rate-and-state parameters, $D_{\mathrm{RS}}$ is the critical slip distance and $f^{*}$ is a reference coefficient of friction at reference slip rate $V^{*}$. The state variable is assumed to evolve according to the aging law (Marone, 1998; Ruina, 1983).

As the fault in the experiment is inactive prior to the fluid stimulation, the modeled fault is not loaded tectonically. Fault slip is thus purely fluid induced, that is, no significant slip would occur without the injection within the time scales considered in the simulations. To initialize the models, we impose shear and normal stresses in agreement with the values reported at the field site prior to the experiment (Guglielmi et al., 2015) and initial state variable values consistent with a dormant, highly healed fault (Text S1; Figures S4-S7). The corresponding initial slip rate is then computed from Equation 4.

\section{Results}

\subsection{Models in Agreement With the Slip Observations During Pressurization}

By first limiting our analysis to the pressurization stage of the experiment (up to $1400 \mathrm{~s}$ ), we find that the observations are equally well reproduced by a family of models. Three representative cases, which we denote lower, intermediate and higher friction models, are shown in Figures 2a-2c and S8-S11 and Table S1. Below, we explain how we constrained these models by examining how the various parameters govern the transitions between the different slip stages and considering the trade-off between friction and fluid pressure. 

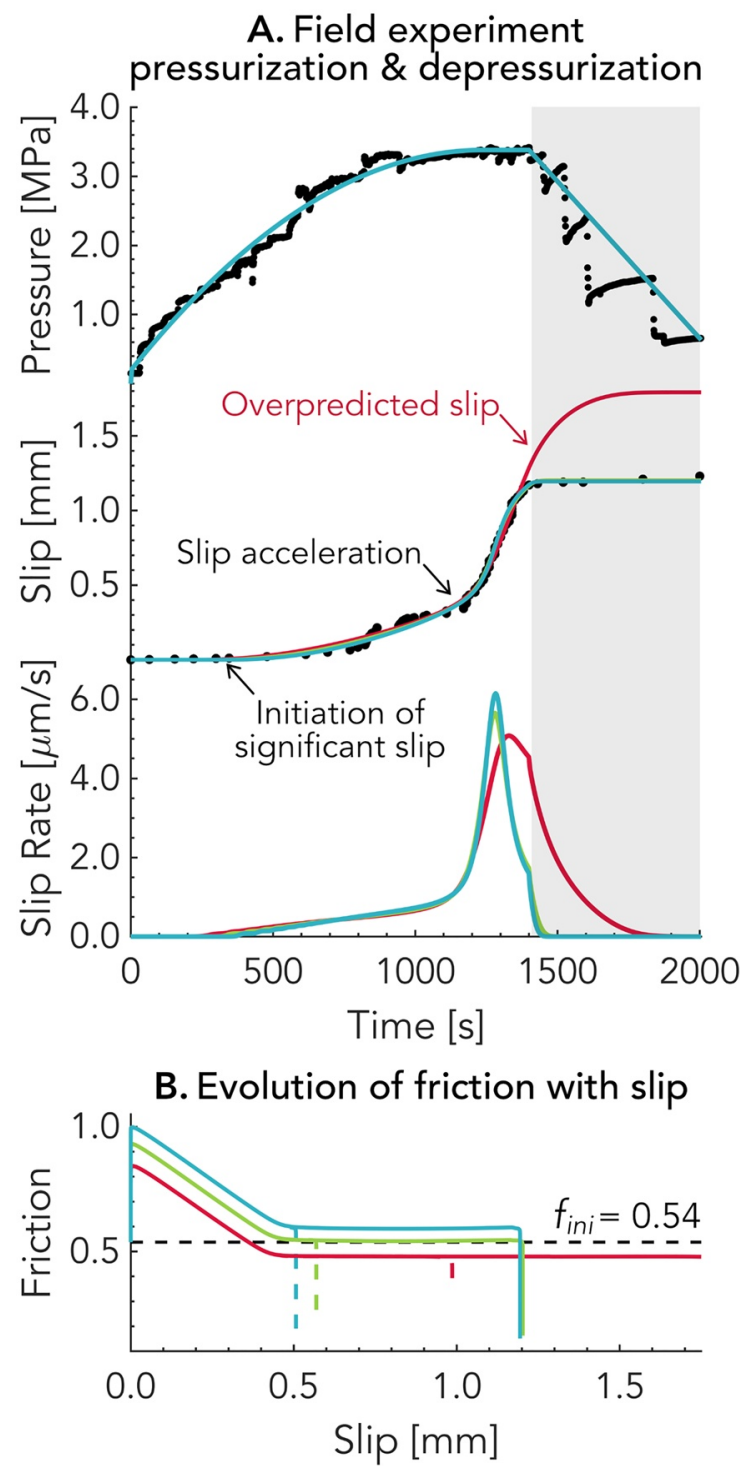

D. More informative depressurization

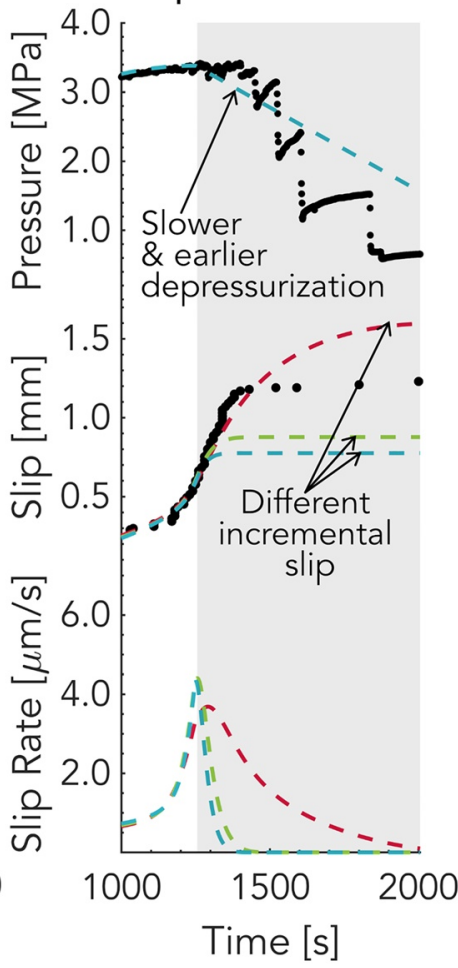

C. Model Properties

$f^{\star} \boldsymbol{\alpha}\left[\mathrm{m}^{2} / \mathrm{s}\right] \quad \mathbf{a}-\boldsymbol{b} \quad \boldsymbol{\theta}_{\text {ini }}[\mathrm{s}]$

$0.48 \quad 0.04-0.001 \quad 1.2 e 12$

$\begin{array}{llll}0.55 & 0.20 & -0.005 & 2.4 e 12\end{array}$

$0.60 \quad 0.85-0.005 \quad 7.0 e 12$

Figure 2. Multiple simulated scenarios match the pressurization stage of the experiment but respond differently to depressurization. (a) Temporal evolution of pore fluid pressure, slip, and slip rate for three model scenarios (solid curves) that reproduce the observations (black dots) during the field-experiment pressurization. (b) Simulated evolution of friction with slip at the injection site; the three scenarios correspond to lower (red), intermediate (green), and higher (blue) residual friction in comparison to the fault prestress (black dashed line). Note that only the intermediate and higher friction faults result in slip consistent with the depressurization part. (c) Key frictional and hydraulic properties of the three scenarios. (d) Similar to (a) but for an improved depressurization: Reducing injection pressure once slip starts to accelerate would allow to distinguish between all three cases, helping to constrain the fault friction properties.

At the beginning of all simulations, slip rates are low and both inertial effects and elastic stress transfers are negligible. Equation 3 is then reduced to:

$$
f(V, \theta)[\sigma-p(x, t)]=\tau_{\text {ini }}
$$

As $p$ increases and $\tau_{\text {ini }}$ remains constant over time, $f$ must increase via growing slip rates in order for Equation 5 to remain true, resulting in a balance between the direct frictional effect and changes in pore pressure (Dublanchet, 2019). Slip rate and friction continue increasing until slip becomes significant at $V \sim 10^{-7} \mathrm{~m} / \mathrm{s}$. The onset of significant slip thus approximately coincides with the maximum friction reached during the simulations (Figures $2 \mathrm{a}, 2 \mathrm{~b}$, and $\mathrm{S} 8$ ). The peak friction, $f^{p}$, can be approximated as: 


$$
f^{p} \sim f^{*}+a \ln \frac{V_{s}}{V^{*}}+b \ln \frac{V^{*} \theta_{\mathrm{ini}}}{D_{\mathrm{RS}}}
$$

where $V_{s}=10^{-7} \mathrm{~m} / \mathrm{s}$. The state variable remains at its initial value, $\theta_{\text {ini }}$, as it has not evolved significantly yet due to negligible slip and short healing time compared to its large initial value. Moreover, because the fluid pressure at the injection site is known at all times, we can relate $f^{p}$ to the timing of slip initiation, $t_{s}$ :

$$
f^{p}=\frac{\tau_{\text {ini }}}{\left[\sigma-p\left(0, t_{s}\right)\right]}
$$

It is thus possible to control $t_{s}$ by computing the corresponding $f^{p}$ with Equation 7, and selecting $f^{*}, a, b$, $\theta_{\text {ini }}$, and $D_{\mathrm{RS}}$ such that Equation 6 is satisfied. The three example models have $t_{s}$ between 300 and $400 \mathrm{~s}$ and $f^{p}$ between 0.84 and 0.99 (Figures $2 \mathrm{~b}$ and S8).

Once significant slip starts accumulating, the fault begins weakening, until it reaches steady state and friction reaches its quasi-static residual value of $f^{r}=f^{*}+(a-b) \ln V / V^{*}$ at the latest stage of the fault pressurization experiment (Figures 2b and S8). As in Dublanchet (2019)'s rate-strengthening models, we find that this transition to steady state is accompanied with a marked acceleration in slip rate (Phase II in Dublanchet, 2019) which we assume to explain the acceleration observed at $1200 \mathrm{~s}$.

The critical slip distance, $\delta_{c}$, over which friction weakens from $f^{p}$ to $f^{r}$ can be approximated as:

$$
\delta_{c} \sim \frac{f^{p}-f^{r}}{b / D_{\mathrm{RS}}}
$$

since $\frac{\partial f}{\partial \delta} \sim \frac{b}{D_{\mathrm{RS}}}$. Furthermore, from elasticity, slip is related to stress drop by:

$$
\Delta \delta \propto \frac{\Delta \tau h}{\mu}
$$

where $h$ is the length of the slipping zone. By equating Equations 8 and 9 at the center of the fault, we can estimate the slipping zone size, $h_{\mathrm{ac}}$, at which steady state is reached and Stage 3 initiates:

$$
h_{\mathrm{ac}} \propto \frac{\mu D_{\mathrm{RS}}}{b} \frac{f^{p}-f^{r}}{\Delta \tau}
$$

Moreover, by choosing $V^{*}$ to be on the same order of magnitude as the fastest slip rate measured during the field experiment $\left(V^{*}=10^{-6} \mathrm{~m} / \mathrm{s}\right)$, we can approximate $f^{r}$ with $f^{*}$, since the contribution of $(a-b) \ln V / V^{*}$ becomes small compared to that of $f^{*}$. Equation 10 can then be rewritten in terms of known parameters as:

$$
h_{\mathrm{ac}} \propto \frac{\mu D_{\mathrm{RS}}}{b} \frac{a \ln \frac{V_{s}}{V^{*}}+b \ln \frac{V^{*} \theta_{\mathrm{ini}}}{D_{\mathrm{RS}}}}{\tau_{\mathrm{ini}}-f^{*}\left[\sigma-p\left(0, t_{\mathrm{ac}}\right)\right]}
$$

where $t_{\mathrm{ac}}$ denotes the onset of Stage 3. For all the simulations presented in this work, we find that adding a prefactor of 3 to Equation 11 provides a good estimate of the slipping zone size at $t_{\text {ac }}$ (Text S2). Remarkably, $h_{\mathrm{ac}}$ only depends on quantities at the injection site. We can thus control the initiation of Stage 3 in our simulations by tuning the model parameters such that the slipping zone reaches length $h_{\mathrm{ac}}$ at $\sim 1200 \mathrm{~s}$, as is the case for our three representative models in Figure 3.

Another critical aspect in these simulations is the balance between friction and the pore pressure forcing. Figures S20-S23 illustrate how the aseismic slip zone grows with decreasing $f^{*}$ and increasing $\alpha$, respectively. In particular, during Stage 3, the spatial extent of the slipping zone with respect to the pressurized 

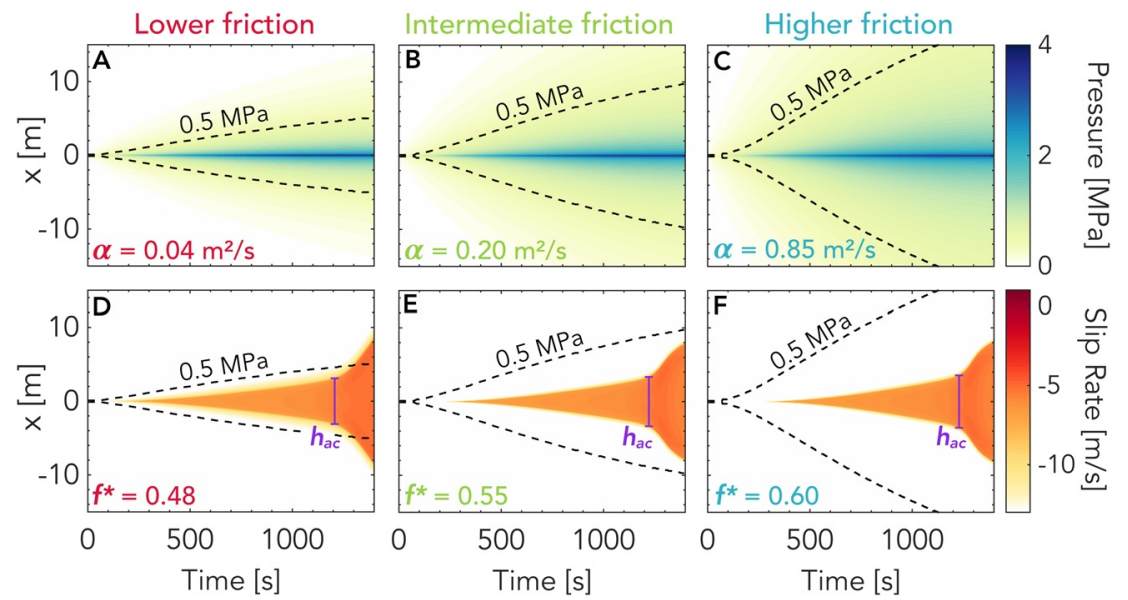

Figure 3. Whether the slipping zone is contained within or outruns the pressurized zone depends on fault friction. Spatial and temporal evolution of $(\mathrm{a}-\mathrm{c})$ pore fluid pressure and (d-f) slip rate for the three scenarios of Figure 2 during pressurization. The purple line shows the estimate $h_{\mathrm{ac}}$ of the slipping zone for the acceleration stage. Black dashed lines indicate the extent of the pressurized zone defined by 0.5 MPa fluid pressure contours. During the pressurization stage, the slipping zone of the lower-friction case outruns the pressurized zone while the intermediate and higher friction cases remain confined to the pressurized zone.

zone as well as the slip rate at the injection site depend on the difference between the residual and initial friction, $f^{r}-f_{\text {ini, }}$ which controls the elastic energy available to drive fault rupture once initiated (Bhattacharya \& Viesca, 2019; Dublanchet, 2019; Galis et al., 2017; Garagash \& Germanovich, 2012) (Figures S19aS19c). Note that this is distinct from the difference between peak and initial friction, $f^{p}-f_{\text {ini }}$ (e.g., Gischig, 2015), which controls the timing of fault reactivation as discussed above.

Given all these considerations, for each diffusion scenario presented in Figure 1b, we find a corresponding frictional model by adjusting $f^{*}$ such that the simulated slip matches the observations during the first two slip stages and produces a sufficiently large slip transient during Stage 3 . To be able to use $f^{r}$ (and hence $f^{*}$ ) values in agreement with the range 0.55-0.65 inferred from laboratory experiments on the grinded fault zone material (Cappa et al., 2019), we set $f_{\text {ini }}$ to $0.54\left(\tau_{\text {ini }}=2.15 \mathrm{MPa}, \sigma=4.00 \mathrm{MPa}\right)$, which is within the uncertainty range of the initial stress measurements. A smaller $f_{\text {ini }}$ would require smaller values of $f^{*}$ outside of this range to obtain the same slip at the injection site. Moreover, the selected values of $f^{*}$ restrict the range of possible values for the term $b \ln V^{*} \theta_{\text {ini }} / D_{\mathrm{RS}}$ in Equation 6 in order for slip to initiate between 300 and $400 \mathrm{~s}$, which in turn restricts factor $\mu D_{\mathrm{RS}} / b$ in Equation 11 in order for Stage 3 to initiate at $1200 \mathrm{~s}$. The factor $\mu D_{\mathrm{RS}}$, which appears in estimates of critical nucleation lengths also needs to be large enough to avoid nucleation of dynamic events within the experimental time (e.g., Rice \& Ruina, 1983; Rubin \& Ampuero, 2005). Finally, we fine tune parameters $a$ and $\theta_{\text {ini }}$ to adjust the slope and timing of the acceleration, respectively. Note that decreasing $a$, while keeping $b$ constant increases the slope of the slip acceleration, due to the (weak) dependence of $f^{r}$ on $(a-b)$, and eventually leads to the nucleation of a dynamic event right at $t_{\text {ac }}$ (Figure S16 and S19d-S19f). This procedure results in a family of models with $f^{*}=0.48-0.60$, $a-b=-0.001-0.005(b=0.016), \theta_{\text {ini }}=1.2 \times 10^{12}-7.0 \times 10^{12} \mathrm{~s}$ and $\alpha=0.04-0.85 \mathrm{~m}^{2} / \mathrm{s}$ that match the slip observations equally well during pressurization.

Although the three models exhibit comparable slip histories at the injection site, they differ in features that were not directly accessible to field observation. In particular, their spatial behaviors differ qualitatively (Figure 3, S9-S11). Defining the pressurized zone with 0.5 MPa pressure contours as in previous works, the lower friction scenario produces an aseismic front that outruns the pressurized region, within $1400 \mathrm{~s}$, as in slip-weakening models (Bhattacharya \& Viesca, 2019) (Figure 3d). By contrast, in the higher friction model, which reproduces the observations equally well, aseismic slip remains confined well within the pressurized area (Figure 3f). Our models demonstrate that slip did not necessarily extend beyond the pressure perturbation during the experiment; that explaining a slip history at a single point in space is a nonunique problem; and that further hydromechanical complexity is not required to explain the observed slip to first 
order. Monitoring fault slip and fluid pressure along the length of the fault, directly with additional probes or remotely with geophysical methods, would help distinguish between these different scenarios and would allow to study additional fault processes such as permeability evolution and inelastic dilatancy (Segall \& Rice, 1995).

\subsection{Distinguishing Between Models With Depressurization}

We find that the depressurization stage of the field experiment, which was not discussed or modeled in previous studies (Bhattacharya \& Viesca, 2019; Cappa et al., 2019; Derode et al., 2015; Guglielmi et al., 2015), contains valuable information on fault properties. In this pressure-reduction stage, the lower friction model features a pronounced delayed slip response that is not observed in the experiment or in the other two cases (Figure 2a). The intermediate and higher friction models, which also have higher hydraulic diffusivities, thus explain the entire set of observations better than the lower friction model. Further discriminating between these two models is not possible with the current data set because by the time depressurization is initiated, the slip rates in these simulations are too low to produce a detectable difference in incremental slip. However, if the injection pressure is decreased more gradually and earlier in the acceleration phase, at which point the intermediate and higher friction scenarios have approximately the same (and higher) slip rate, the three scenarios lead to diverging levels of incremental slip (Figure 2d). As we only investigate a limited portion of the rate-and-state parameter space in this study, we cannot conclude that timely depressurization can uniquely discriminate between all possible frictional scenarios. However, it is clear that timely depressurization can provide additional constraints on the frictional and hydromechanical properties of fault zones.

In addition to fitting the entire set of slip observations better, models with $f^{*}$ of 0.55 and 0.60 are also more consistent with the range of residual friction values of 0.55-0.65 derived from laboratory experiments on grinded fault gouge (Cappa et al., 2019). Moreover, the initial fault conditions implied by these higher friction cases are fully consistent with those of a dormant fault whereas the low-friction case is not (Text S1). Our preferred model for the site of the injection experiment is thus a rate-weakening fault with $0.55<f^{*}$ $<0.60,0.20<\alpha<0.85 \mathrm{~m}^{2} / \mathrm{s}, a=0.011$ and $b=0.016$. This is in contrast to the original Guglielmi et al. (2015) study in which the authors inferred a rate-strengthening fault from a spring-slider model with permeability enhancement. Within the limited parameter space that we explored through the procedure outlined in Section 3.1, we could only find rate-strengthening models with relatively low $f^{*}$ and hence ones that only match the pressurization stage of the experiment (Figure S24). It is possible that there are rate-strengthening models that match the entire slip history that we have not considered here but that would not change our conclusions that the field measurements can be matched with multiple friction scenarios and that the depressurization stage provides further constraints than pressurization alone.

\subsection{Diverging Fault Stability With Sustained Injection}

Modeling what would have happened if the fluid injection had continued for longer highlights why distinguishing between the three qualitatively different scenarios identified in this study is crucial. In response to an extended constant-pressure injection (Figure 4, Figures S3, S25-S27), the low-friction fault nucleates an earthquake almost immediately, while the intermediate and higher friction faults decelerate and continue slipping aseismically before eventually transitioning to seismic slip rates. Once a seismic rupture initiates, whether it is self-arrested or runaway depends on the dynamic residual friction, $f^{d}$, which is generally slightly lower than $f^{r}$ (Galis et al., 2017; Garagash \& Germanovich, 2012). If $f^{d}<f_{\text {ini, }}$ as in the low- and intermediate-friction cases (Figure $4 \mathrm{~b}$ ), the rupture may release enough elastic energy to propagate beyond the fluid-affected regions and would only be stopped by less favorably stressed fault patches, geometrical barriers, or more stable materials not present in the current model (Figures 4c and 4d). Such runaway ruptures may be preceded by smaller ruptures or aseismic slip transients (Figures S15 and S19a); indeed, in fracture mechanics models (Galis et al., 2017), the transition to runaway rupture requires a certain balance between fluid pressurization and background stress to be reached. If $f^{d}>f_{\text {ini }}$, as in the high-friction case, the rupture self-arrests once out of the pressurized zone (Figure 4e). For low- to intermediate-friction faults, the maximum expected earthquake magnitude, $M_{\max }$, is thus controlled by hydromechanical 

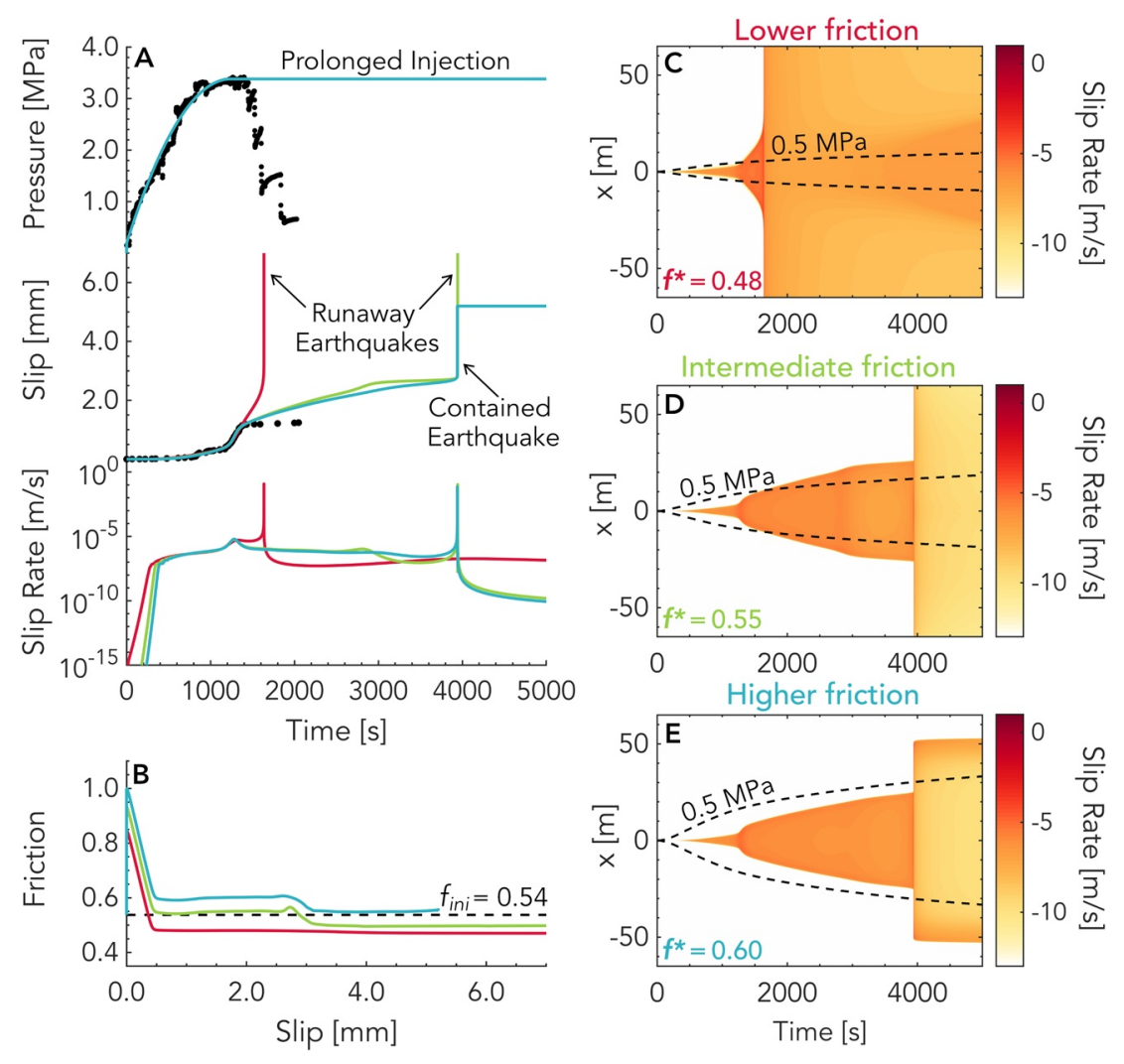

Figure 4. Prolonged injection reveals the diverging stability of the different fault models. Same as Figure 2 (a-b) and Figure 3 (c-e) but for a longer injection scenario, keeping the pressure at the center of the fault constant past $1400 \mathrm{~s}$ instead of decreasing it. The low-friction case (red in a, c) produces a runaway earthquake rupture much sooner than the intermediate-friction case (green in a, d), while the higher friction case (blue in a, e), which is consistent with most known information about the fault, results in a self-arresting earthquake confined to the pressurized zone (blue).

and geometrical fault properties as opposed to injection attributes (e.g., cumulative volume injected) (Galis et al., 2017; Gischig, 2015; McGarr, 2014; van der Elst et al., 2016). For example, varying the injection rate in our simulations does not alter the event size (Figure S28). In the intermediate-friction case, the fault ultimately undergoes a runaway earthquake despite having stably released energy for over an hour, thus demonstrating that aseismic slip does not signify an absence of earthquake hazard. Fortunately, comparing the depressurization and prolonged injection scenarios reveals that reducing the injection pressure might be sufficient to suppress earthquake nucleation at the injection site. The lower the friction on the fault, the faster the rate of this depressurization needs to be (Figure S29). Note, however, that earthquakes could still be triggered by aseismic slip itself on more unstable heterogeneities away from the injection site (Eyre et al., 2019; Guglielmi et al., 2015).

\section{Discussion and Conclusions}

To summarize, our modeling of a fluid-injection experiment into a fault zone reveals that the difference between fault prestress and quasistatic or dynamic fault friction controls whether slip is confined to the fluid-affected zone or outruns it. We find that: (a) multiple scenarios with different hydrologic assumptions and friction levels are consistent with the measured slip at the injection site during the pressurization phase; (b) the low-friction scenario, in which slow slip outruns the pressurized region is inconsistent with slip during the depressurization phase; and (c) the high-friction scenario, in which the slipping zone is well confined within the pressurized region, is most consistent with the full range of information from the experiment, including the fault behavior during fault depressurization and laboratory friction measurements on the materials from the fault zone. Key hydromechanical parameters such as the difference between 
quasistatic friction and initial normalized prestress, $f^{r}-f_{\text {ini, }}$, the rate dependence of friction, $a-b$, and the hydraulic diffusivity, $\alpha$, exercise a first-order control on the stability and spatial extent of a fault response to fluid injections. Further constraining these parameters is thus critical for seismic hazard management. In the geoenergy industry, test injections with timely depressurization and spatiotemporal monitoring of fluid pressure and aseismic slip could be performed prior to exploitation to ensure that there are no low-friction faults nearby. Our findings show that augmenting fault-pressurization experiments with suitably designed depressurization phases and multiple monitoring locations along the fault could provide invaluable insight into the physics of both induced and natural earthquakes (Savage et al., 2017) and friction properties of dormant faults. These more advanced injection experiments and corresponding modeling work will potentially be able to assess the effects and relative importance of additional mechanisms such as poroelastic stresses (Deng et al., 2016; Goebel et al., 2017; Segall \& Lu, 2015), slip-induced dilatancy (Cappa et al., 2019; Segall \& Rice, 1995), bulk fluid diffusion, enhanced dynamic weakening, and material heterogeneities (e.g., Eyre et al., 2019).

\section{Conflict of Interest}

The authors declare no competing interests.

\section{Data Availability Statement}

The data supporting the analysis and conclusions are given in Figures and Tables, in the main text, and supplementary materials. Model outputs and the experimental data from the Guglielmi et al. (2015) field experiment are accessible through the CaltechDATA repository (https://data.caltech.edu/records/1891). Data from the field experiment as reported in Guglielmi et al. (2015) can be found in the supplementary materials.

\section{Acknowledgments}

This study was supported by the National Science Foundation (Grants EAR 1151926 and EAR 1724686), the NSF-IUCRC Center for Geomechanics and Mitigation of Geohazards (projects GMG-4.1, GMG-4.2), the National Sciences and Engineering Research Council of Canada (PGSD-3-5170782018), and the French government through the UCAJEDI Investments in the Future project managed by the $\mathrm{Na}$ tional Research Agency (ANR) with the reference number ANR-15-IDEX-01. The computations presented here were conducted on the Caltech High Performance Cluster. The authors thank Jean-Philippe Avouac, Pathikrit Bhattacharya, Yves Guglielmi, and Robert C. Viesca for helpful discussions as well as Valère Lambert and Oliver Stephenson for help with the simulation code. We would also like to thank the editor Germán Prieto as well as Eric Dunham and one anonymous reviewer for their constructive comments which have led to an improved manuscript.

\section{References}

Bhattacharya, P., \& Viesca, R. C. (2019). Fluid-induced aseismic fault slip outpaces pore-fluid migration. Science, 364, 464-468. https://doi. org/10.1126/science.aaw7354

Cappa, F., Scuderi, M. M., Collettini, C., Guglielmi, Y., \& Avouac, J.-P. (2019). Stabilization of fault slip by fluid injection in the laboratory and in situ. Science Advances, 5, eaau4065. https://doi.org/10.1126/sciadv.aau4065

Cornet, F. H., Helm, J., Poitrenaud, H., \& Etchecopar, A. (1997). Seismic and Aseismic Slips Induced by Large-scale Fluid Injections. In Seismicity associated with mines, reservoirs and fluid injections (pp. 563-583). Basel: Birkhäuser Basel. https://doi. org/10.1007/978-3-0348-8814-1_12

Deng, K., Liu, Y., \& Harrington, R. M. (2016). Poroelastic stress triggering of the December 2013 Crooked Lake, Alberta, induced seismicity sequence. Geophysical Research Letters, 43, 8482-8491. https://doi.org/10.1002/2016GL070421

Derode, B., Guglielmi, Y., De Barros, L., \& Cappa, F. (2015). Seismic responses to fluid pressure perturbations in a slipping fault. Geophysical Research Letters, 42, 3197-3203. https://doi.org/10.1002/2015GL063671

Dieterich, J. H. (1979). Modeling of rock friction: 1. Experimental results and constitutive equations. Journal of Geophysical Research, 84, 2161. https://doi.org/10.1029/JB084iB05p02161

Dieterich, J. H. (2007). Applications of rate- and state-dependent friction to models of fault slip and earthquake occurrence. Treatise on geophysics (pp. 107-129). https://doi.org/10.1016/B978-044452748-6.00065-1

Dublanchet, P. (2019). Fluid driven shear cracks on a strengthening rate-and-state frictional fault. Journal of the Mechanics and Physics of Solids, 132, 103672. https://doi.org/10.1016/j.jmps.2019.07.015

Duboeuf, L., De Barros, L., Cappa, F., Guglielmi, Y., Deschamps, A., \& Seguy, S. (2017). Aseismic motions drive a sparse seismicity during fluid injections into a fractured zone in a carbonate reservoir. Journal of Geophysical Research: Solid Earth, 122, 8285-8304. https://doi. org/10.1002/2017JB014535

Ellsworth, W. L. (2013). Injection-induced earthquakes. Science, 341, 1225942. https://doi.org/10.1126/science.1225942

Eyre, T. S., Eaton, D. W., Garagash, D. I., Zecevic, M., Venieri, M., Weir, R., \& Lawton, D. C. (2019). The role of aseismic slip in hydraulic fracturing-induced seismicity. Science Advances, 5, eaav7172. https://doi.org/10.1126/sciadv.aav7172

Galis, M., Ampuero, J. P., Mai, P. M., \& Cappa, F. (2017). Induced seismicity provides insight into why earthquake ruptures stop. Science Advances, 3, eaap7528. https://doi.org/10.1126/sciadv.aap7528

Garagash, D. I., \& Germanovich, L. N. (2012). Nucleation and arrest of dynamic slip on a pressurized fault. Journal of Geophysical Research, 117. https://doi.org/10.1029/2012JB009209

Gischig, V. S. (2015). Rupture propagation behavior and the largest possible earthquake induced by fluid injection into deep reservoirs. Geophysical Research Letters, 42, 7420-7428. https://doi.org/10.1002/2015GL065072

Goebel, T. H. W., \& Brodsky, E. E. (2018). The spatial footprint of injection wells in a global compilation of induced earthquake sequences. Science, 361, 899-904. https://doi.org/10.1126/science.aat5449

Goebel, T. H. W., Weingarten, M., Chen, X., Haffener, J., \& Brodsky, E. E. (2017). The 2016 Mw5.1 Fairview, Oklahoma earthquakes: Evidence for long-range poroelastic triggering at $>40 \mathrm{~km}$ from fluid disposal wells. Earth and Planetary Science Letters, 472, 50-61. https://doi.org/10.1016/J.EPSL.2017.05.011 
Grigoli, F., Cesca, S., Priolo, E., Rinaldi, A. P., Clinton, J. F., Stabile, T. A., et al. (2017). Current challenges in monitoring, discrimination, and management of induced seismicity related to underground industrial activities: A European perspective. Reviews of Geophysics, 55, 310-340. https://doi.org/10.1002/2016RG000542

Guglielmi, Y., Cappa, F., Avouac, J.-P., Henry, P., \& Elsworth, D. (2015). Seismicity triggered by fluid injection-induced aseismic slip. Science, 348, 1224-1226. https://doi.org/10.1126/science.aab0476

Lapusta, N., Rice, J. R., Ben-Zion, Y., \& Zheng, G. (2000). Elastodynamic analysis for slow tectonic loading with spontaneous rupture episodes on faults with rate- and state-dependent friction. Journal of Geophysical Research, 105, 23765-23789. https://doi. org/10.1029/2000JB900250

Marone, C. (1998). Laboratory-derived friction laws and their application to seismic faulting. Annual Review of Earth and Planetary Sciences, 26. https://doi.org/10.1146/annurev.earth.26.1.643

McGarr, A. (2014). Maximum magnitude earthquakes induced by fluid injection. Journal of Geophysical Research: Solid Earth, 119, 10081019. https://doi.org/10.1002/2013JB010597

McGarr, A., Bekins, B., Burkardt, N., Dewey, J., Earle, P., Ellsworth, W., et al. (2015). Coping with earthquakes induced by fluid injection. Science, 347, 830-831. https://doi.org/10.1126/science.aaa0494

Noda, H., \& Lapusta, N. (2013). Stable creeping fault segments can become destructive as a result of dynamic weakening. Nature, 493, 518-521. https://doi.org/10.1038/nature11703

Rice, J. R., \& Ruina, A. L. (1983). Stability of steady frictional slipping. Journal of Applied Mechanics, 50, 343-349. https://doi. org/10.1115/1.3167042

Rubin, A. M., \& Ampuero, J.-P. (2005). Earthquake nucleation on (aging) rate and state faults. Journal of Geophysical Research, 110. https:// doi.org/10.1029/2005JB003686

Ruina, A. (1983). Slip instability and state variable friction laws. Journal of Geophysical Research, 88, 10359-10370. https://doi.org/10.1029/ JB088iB12p10359

Savage, H. M., Kirkpatrick, J. D., Mori, J. J., Brodsky, E. E., Ellsworth, W. L., Carpenter, B. M., et al. (2017). Scientific exploration of induced seismicity and stress (SEISMS). Scientific Drilling, 23, 57-63. https://doi.org/10.5194/sd-23-57-2017

Scotti, O., \& Cornet, F. H. (1994). In situ evidence for fluid-induced aseismic slip events along fault zones. International Journal of Rock Mechanics and Mining Science \& Geomechanics Abstracts, 31, 347-358. https://doi.org/10.1016/0148-9062(94)90902-4

Segall, P., \& Lu, S. (2015). Injection-induced seismicity: Poroelastic and earthquake nucleation effects. Journal of Geophysical Research: Solid Earth, 120, 5082-5103. https://doi.org/10.1002/2015JB012060

Segall, P., \& Rice, J. R. (1995). Dilatancy, compaction, and slip instability of a fluid-infiltrated fault. Journal of Geophysical Research, 100, 22155-22171. https://doi.org/10.1029/95JB02403

van der Elst, N. J., Page, M. T., Weiser, D. A., Goebel, T. H. W., \& Hosseini, S. M. (2016). Induced earthquake magnitudes are as large as (statistically) expected. Journal of Geophysical Research: Solid Earth, 121, 4575-4590. https://doi.org/10.1002/2016JB012818

Wei, S., Avouac, J.-P., Hudnut, K. W., Donnellan, A., Parker, J. W., Graves, R. W., et al. (2015). The 2012 Brawley swarm triggered by injection-induced aseismic slip. Earth and Planetary Science Letters, 422, 115-125. https://doi.org/10.1016/j.epsl.2015.03.054

\section{References From the Supporting Information}

Dieterich, J. H. (1992). Earthquake nucleation on faults with rate-and state-dependent strength. Tectonophysics, 211, 115-134. https://doi. org/10.1016/0040-1951(92)90055-B

Helmstetter, A., \& Shaw, B. E. (2009). Afterslip and aftershocks in the rate-and-state friction law. Journal of Geophysical Research, 114 https://doi.org/10.1029/2007JB005077

Uenishi, K., \& Rice, J. R. (2003). Universal nucleation length for slip-weakening rupture instability under nonuniform fault loading. Journal of Geophysical Research, 108(B1). https://doi.org/10.1029/2001JB001681

Viesca, R. C., \& Rice, J. R. (2012). Nucleation of slip-weakening rupture instability in landslides by localized increase of pore pressure. Journal of Geophysical Research, 117. https://doi.org/10.1029/2011JB008866 